\title{
The effect of an infrared divergent quark-antiquark interaction kernel on other Green functions
}

\author{
Reinhard Alkofer* \\ Institut für Physik, Karl-Franzens-Universität Graz, Universitätsplatz 5, 8010 Graz, Austria \\ E-mail: reinhard.alkofer@uni-graz.at
}

\section{Mario Mitter}

Universität Heidelberg, Institut für Theoretische Physik, Philosophenweg 16, D-69120

Heidelberg, Germany

E-mail: mario.mitter@thphys.uni-heidelberg.de

\begin{abstract}
The $n$-point Green functions of Landau gauge QCD are systematically investigated in a DysonSchwinger approach assuming a static linearly rising potential between fundamental color charges. Besides quarks also scalar matter in the fundamental representation is considered. Starting from the hypothesis of an $1 / k^{4}$ infrared divergent matter-antimatter vertex restrictions on the general color tensor structure of this divergence are derived. Consequences for the other fourpoint functions of QCD, resp., scalar QCD, are presented. Hereby Casimir scaling is found. It is shown that possible singular contributions to the three-point functions vanish due to cancellations within the color algebra. On the other hand, higher $n$-point functions inherit the infrared singularity of the matter-antimatter vertex in certain color channels. The presented results show that linear confinement is consistently possible in a Greens function approach, however, at the expense that the decoupling theorem is circumvented by infrared singularities.
\end{abstract}

Xth Quark Confinement and the Hadron Spectrum

8-12 October 2012

TUM Campus Garching, Munich, Germany

\footnotetext{
* Speaker.
} 


\section{Motivation}

The title of this conference series is Quark Confinement and the Hadron Spectrum. Over the last eighteen years this conference series has documented the impressive progress on nonperturbative QCD and hadron physics which has been achieved by a wealth of methods. Nevertheless, it is fair to say that our current understanding of quark confinement is far from being satisfactory. In this talk another facet is added to the discussion. It is based on the so-called quenched approximation and the then resulting linearly rising static potential in between infinitely heavy, i.e., non-dynamical, quarks. Such a potential has been impressively verified by numerous lattice calculations in which the Wilson loop has been computed and its area law related to the linearly rising potential has been extracted with high precision.

In this talk the question whether and how such a linearly rising static potential can be encoded in the $n$-point Greens functions of quenched QCD will be discussed. Although the corresponding investigation is still far from being complete some interesting positive answers along with some puzzling facts will result. But as a first step a disclaimer is in order. Due to the exponentiation of the gluon field the Wilson loop depends on infinitely many $n$-point functions. Therefore the observed area law of the Wilson loop does not provide a compelling reason why a finite set of $n$-point functions should already lead to confinement in the sense of a linearly rising potential. On the other hand, one can show that an infrared singular quark interaction can provide such a linearly rising potential. The typical starting point for such an investigation (also taken here) is the hypothesis that some tensor components of the quark four-point function diverges like $1 / k^{4}$ for small exchanged momentum $k$ ( $c f$., Fig. 1). If such an infrared divergence is properly regularized [1] and then Fourier transformed it leads, in the non-relativistic limit, to a heavy quark potential with a term linear in the distance $r$, i.e., to the anticipated linearly rising potential. This provides an example how confinement can be encoded already in a single $n$-point function.

In the following we will only briefly summarize the main line of argumentation and then the consequences for other Greens functions which are implied by an infrared singular four-point function, a detailed discussion can be found in Ref. [2].

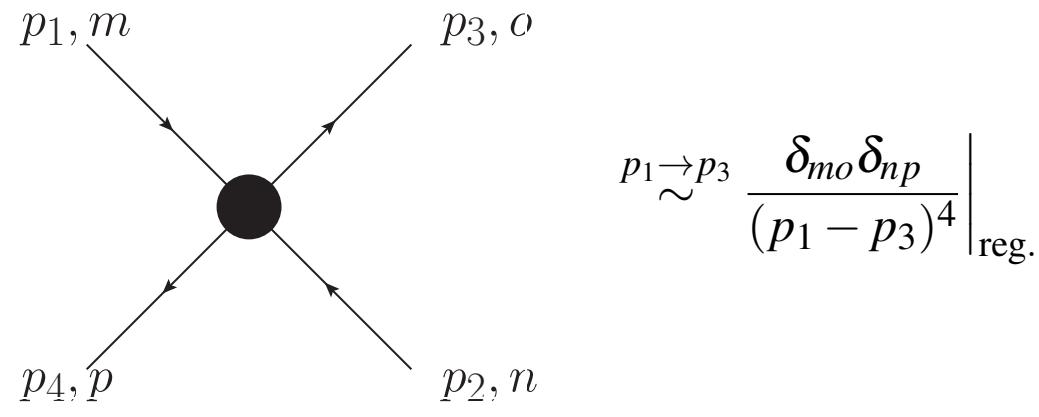

Figure 1: An illustration of the color indices and the momentum dependence of the matter four-point function as well as the color tensor structure for the assumed infrared singularity. 


\section{A remark on Landau gauge QCD Greens functions}

As Greens functions of colored fields are gauge dependent quantities one might even speculate whether in different gauges the situation is qualitatively different. Of course, as we wish to investigate confinement in a functional approach we would choose a gauge where confinement is contained in a finite set of $n$-point functions. As by now there is no hint which gauge might be best suited for our planned study we simply choose the gauge in which the Greens functions are best known, the Landau gauge. Also in this respect we cannot be sure that in the non-perturbative domain Landau gauge is uniquely defined, see, e.g., Ref. [3] and references therein.

Actually, early investigations of the Landau gauge gluon propagator were exploring the possibility whether the $1 / k^{4}$ infrared singularity might already exist in this propagator, see, e.g., Refs. $[4,5]$ and references therein. By now it is clear that the Landau gauge gluon propagator is suppressed in the infrared and not enhanced ${ }^{1}$ : The Landau gauge gluon is confined and not confining. On the other hand, in the so-called scaling solution of Dyson-Schwinger and Functional Renormalization Group Equations the quark-gluon vertex can be infrared singular such that the four-point function assumes the $1 / k^{4}$ singularity [7]. In this respect it is interesting to note that such an infrared singularity provides a description of the $U_{A}(1)$ anomaly within a Greens function approach [8]. Taken all this together motivates our study of the possibility of an $1 / k^{4}$ singularity in the matter four-point function in Landau gauge QCD as well as an investigation of the consequences for all other Greens functions. ${ }^{2}$

\section{Assuming an infrared divergent four-point function}

As detailed above the hypothesis to be tested in this investigation is whether the four-point function can be maximally infrared singular without causing any contradiction when plugged in into the equations for an arbitrary Greens function. Here we will focus on the use of DysonSchwinger equations for this purpose. As the derivation of Dyson-Schwinger equations for higher $n$-point functions by hand ranges from tedious to impossible corresponding tools have been employed [10]. A good example for the complexity of such a Dyson-Schwinger equation is already the one for the quark four-point function, see Fig. 2 for a diagrammatical representation.

As we are interested in the role of infrared divergent terms in the confinement mechanism special emphasis will be laid on the color structure of potentially infrared divergent terms. The spinor property of quarks is neglected in the following discussion as fundamentally charged matter is also expected to be confined. In the calculations we employed as gauge group $\mathrm{SU}(N)$.

\footnotetext{
${ }^{1}$ A discussion of the infrared behaviour of the Landau gauge gluon propagator can be found, e.g., in the reviews [6] and references therein. In other gauges one might have infrared enhanced and infrared suppressed components of the gluon propagator. Examples are Coulomb gauge with an infrared enhanced temporal and an infrared suppressed spatial gluon propagator, or maximally Abelian gauge with an infrared enhanced color-diagonal and an infrared suppressed color-off-diagonal gluon propagator.

${ }^{2}$ Although hadrons as colorless bound states are in the focus of interest when studying the four-point functions in the meson and the six-point functions in the baryon channels there is also some reason to study colored higher $n$-point functions, e.g., to look for negative-metric and zero-norm bound states containing ghosts to complete the nonperturbative BRST quartets [9].
} 


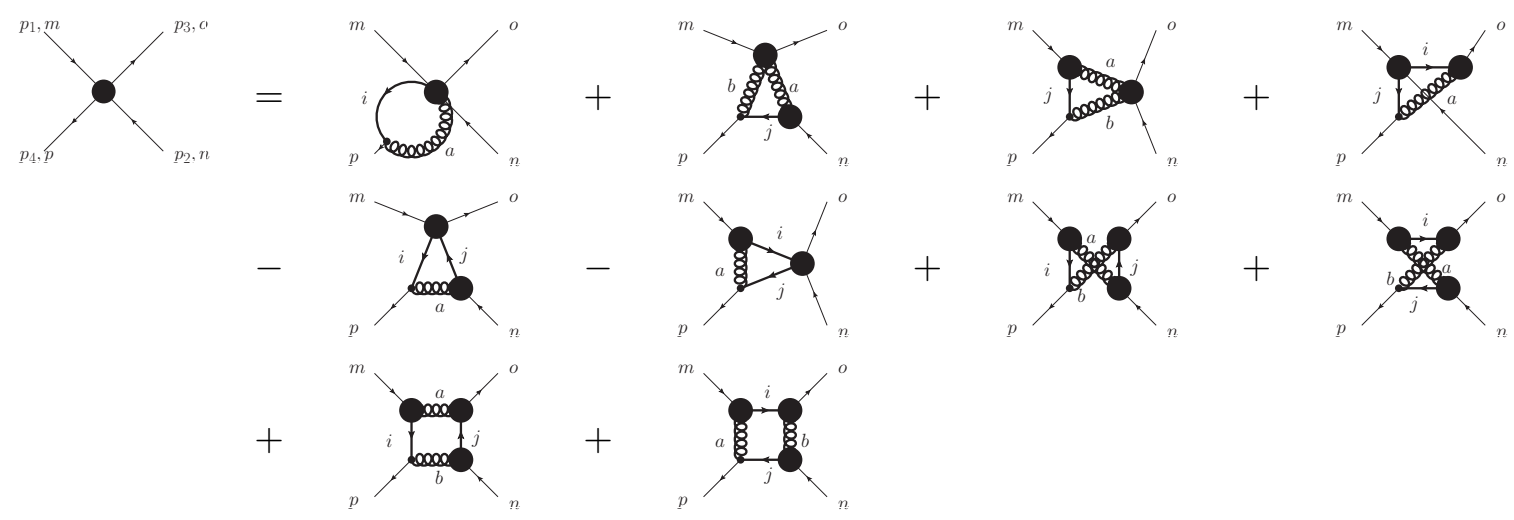

Figure 2: The Dyson-Schwinger for the quark four-point function.

At first sight the Dyson-Schwinger equation for the matter four-point function causes no immediate contradiction. But a restriction arises when considering the equation for the matter-gluon vertex which would receive a singular contribution

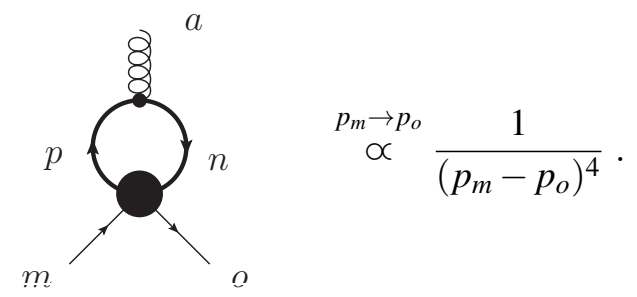

Feeding the resulting infrared behavior of this vertex into the equations for $n \geq 4$ leads to divergencies stronger than the initially assumed $1 / k^{4}$ behavior. In case of the quark four-point functions the infrared singularity of the box diagrams would exceed the initially assumed divergent behavior by infrared power counting.

Therefore the boundedness of higher $n$-point functions to a maximal infrared singularity of the $1 / k^{4}$ type requires a less singular matter-gluon vertex which in turn implies that the infrared singularity can only be present in one of the two possible color structures, the divergent one is shown in Fig. 1. In this respect it is interesting to note that one-gluon exchange fails to reproduce this color structure. The fact that in this way one can exclude an one-gluon exchange as source for confinement comes as no surprise: Such a type of confinement would lead to the analogue of van-der-Waals forces [11] which are not seen in nature.

By identifying corresponding diagrams one can more or less straightforwardly demonstrate that in case of a $1 / k^{4}$ singularity in the color non-singlet channel of the matter four-point function all other four-point functions (i.e., the four-gluon vertex as well as the ghost-gluon, matter-ghost and matter-gluon scattering kernels) acquire an analogous infrared singularity in respective specific color channels. For example the quark-gluon four-point function receives a contribution 


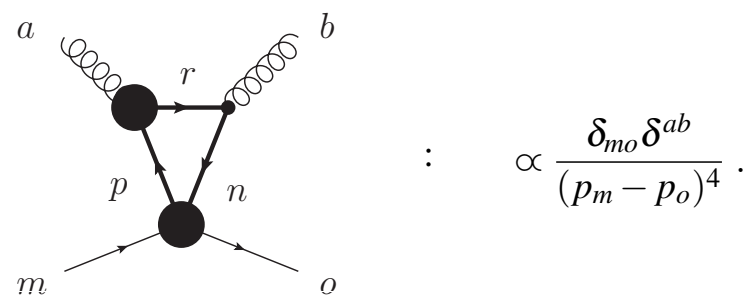

Again straightforward application of identities of the $\mathrm{SU}(N)$ color algebra can be used to check that these newly appearing singularities are not forwarded to the quark-gluon vertex or other three-point functions

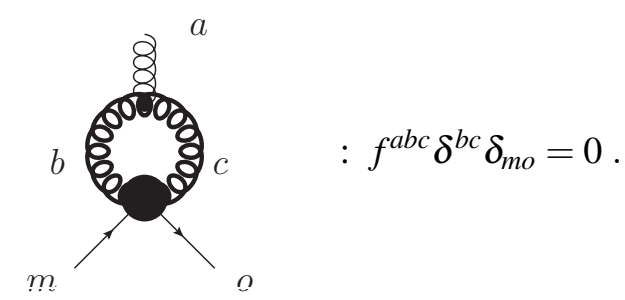

Even higher $n$-point functions can be treated when factorizing the tensor structures with the help of diagrammatic methods [12]. This allows to map the color weights of diagrams of $(n+m)$ point functions to the color weights of diagrams of $n$ - and $m$-point functions. In this way one can inductively derive how the starting hypothetical $1 / k^{4}$ infrared singularity in the four-point function propagates through the tower of all higher Greens functions. Thus one can conclude that if the starting hypothesis is true all higher $n$-point functions contain contributions $\propto 1 / k^{4}$ with $k$ being the momentum transfer between two colored clusters.

\section{Discussion}

One non-trivial result of this analysis is the fact that propagators and three-point functions are protected by systematic cancelations in the color algebra against infrared singularities. These, if existed, would in turn lead to unaccceptably strong infrared singularities in the $n \geq 4$-point functions. We have verified these cancelations if the color group is $\mathrm{SU}(N)$. It would be interesting to check whether the algebras of other gauge groups as, e.g., $\mathrm{SO}(N)$ or $G_{2}$, allow for the same conclusion.

The second, although not so astonishing, result which is nevertheless worth mentioning is that an one-gluon exchange cannot be the (only) source of an infared singularity. As already metioned the old picture of infrared slavery by gluon exchange is anyhow challenged by contradictions to phenomelogy and lattice results [11].

Third, one can check by explicit inspection of the color weights that the assumed confining $1 / k^{4}$ singularity in the four-point function implies Casimir scaling (which has been unambigously identified in lattice calculations) for the confining potential between colored clusters. If for example the singularity originates from the diagrams containing the quark-gluon four-point function a simple calculation yields singular contributions 


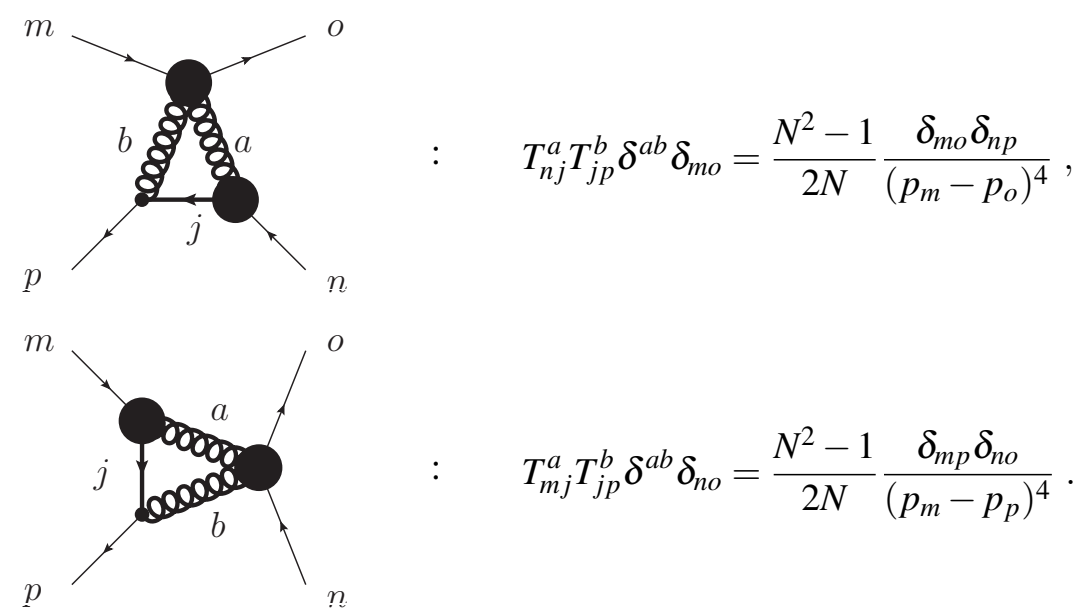

For a general representation of the gauge group these diagrams result in a singular contribution proportional to the quadratic Casimir invariant. Similarly other diagram contributing to the quark four-point function can be either excluded as sources of the singularity or yield the correct Casimir scaling factor.

In addition, the color channels of the infrared singularities in higher $n$-point functions are in accordance with arguments how the cluster decomposition property might fail in QCD. Proper regularization of the $1 / k^{4}$ singularity in the higher $n$-point functions results in a $\delta$-function type contribution which in turn relates to a possible way of violating cluster decomposition.

Fourth, and actually most surprising, the presented results demonstrate how the decoupling theorem [13] is circumvented by infrared singularities: One very heavy fundamental charge, once introduced into the theory, will by virtue of the $1 / k^{4}$ infrared singularity in its four-point function induce a change in the infrared behavior of Greens functions in the Yang-Mills sector.

\section{Summary and Outlook}

To summarize, we remark that the assumption of a confining infrared singularity in the mattermatter scattering kernel leads to several wanted features. Certainly not all possible consequences of a $1 / k^{4}$ singularity in the quark four-point function have been investigated in this first study because only the color structures have been taken into account. The starting hypothesis of a maximal singularity has been found consistent if it appears in a special color structure that cannot be reproduced by the exchange of one gluon. This singularity is directly propagated to the other four- and higher $n$-point functions. Although singularities are induced in all $n$-point functions with $n \geq 4$ the three-point functions and propagators are protected. And, most important, singularities appear whenever the exchanged momentum between colored subsets of legs of a $n$-point function vanishes. In addition, a simple explanation of Casimir scaling is provided.

Already one four-point function even when corresponding to a very heavy fundamentally charged field could propogate such a singularity to other Yang-Mills or matter correlation functions. Although this is no contradiction to the decoupling theorem, which holds only in the absence of singularities, it is nevertheless intriguing. Of course, whether there is then no decoupling, or more precisely, how then the decoupling of infinitely heavy fundamental charges in gauge-invariant quantities takes place certainly deserves further studies. 
Also other related and yet not understood aspects come immediately to one's mind: How do potential van-der-Waals forces cancel? What about $N$-ality and string breaking for adjoint charges? Is there a direct relation to dynamical chiral symmetry breaking?

\section{Acknowledgements}

We are grateful to the organizers of the Xth Quark Confinement and the Hadron Spectrum conference for all their efforts which made this extraordinary event possible.

During the course of this work MM was funded by the Austrian Science Fund, FWF, through the Doctoral Program on Hadrons in Vacuum, Nuclei, and Stars (FWF DK W1203-N16).

\section{References}

[1] D. Gromes, Z. Phys. C 11 (1981) 147.

[2] M. Mitter and R. Alkofer, in preparation; see also: Chapter 3 of M. Mitter, PhD thesis, U. Graz, November 2012.

[3] A. Maas, these proceedings [arXiv:1301.2965 [hep-th]]; Phys. Lett. B 689 (2010) 107 [arXiv:0907.5185 [hep-lat]].

[4] S. Mandelstam, Phys. Rev. D 20 (1979) 3223.

[5] N. Brown and M. R. Pennington, Phys. Rev. D 39 (1989) 2723.

[6] R. Alkofer and L. von Smekal, Phys. Rept. 353 (2001) 281 [arXiv:hep-ph/0007355]; C. S. Fischer, J. Phys. G 32 (2006) R253 [hep-ph/0605173]; D. Binosi and J. Papavassiliou, Phys. Rept. 479 (2009) 1 [arXiv:0909.2536 [hep-ph]]; A. Maas, Phys. Rept., in press (available online) [arXiv:1106.3942 [hep-ph]].

[7] R. Alkofer, C. S. Fischer, F. J. Llanes-Estrada and K. Schwenzer, Annals Phys. 324 (2009) 106 [arXiv:0804.3042 [hep-ph]]; M. Hopfer, A. Windisch and R. Alkofer, PoS CONFINEMENTX (2013) 073 [arXiv:1301.3672 [hep-ph]].

[8] R. Alkofer, C. S. Fischer and R. Williams, Eur. Phys. J. A 38 (2008) 53 [arXiv:0804.3478 [hep-ph]].

[9] N. Alkofer and R. Alkofer, PoS CONFINEMENTX (2013) 282 [arXiv:1301.5292 [hep-th]]; Phys. Lett. B 702 (2011) 158 [arXiv:1102.2753 [hep-th]].

[10] M. Q. Huber and J. Braun, Comput. Phys. Commun. 183 (2012) 1290 [arXiv:1102.5307 [hep-th]]; M. Q. Huber and M. Mitter, Comput. Phys. Commun. 183 (2012) 2441 [arXiv:1112.5622 [hep-th]]; R. Alkofer, M. Q. Huber and K. Schwenzer, Comput. Phys. Commun. 180 (2009) 965 [arXiv:0808.2939 [hep-th]].

[11] J. Greensite, “An introduction to the confinement problem,” Lect. Notes Phys. 821 (2011) 1.

[12] P. Cvitanovic, “Group theory: Birdtracks, Lie's and exceptional groups,” NORDITA lecture notes and Princeton University Press (2008).

[13] T. Appelquist and J. Carazzone, Phys. Rev. D 11 (1975) 2856. 